\title{
A Design of a PI Compensator for a Bidirectional DC-DC Converter in a DC Distributed Power System
}

\author{
Joonmin Lee*, Bong Jun Seok*, Jae Du La** and Young Seok Kim***
}

\begin{abstract}
The Voltage Bus Conditioner(VBC) is a bidirectional DC-DC converter for damping the instability and any transients of the bus voltage in a DC Distributed Power System(DPS). In this paper, a PI controller for the VBC has been designed for the frequency domain. The proposed PI controller not only dampens the bus transients, but also keeps the storage voltage level. Simulation by Matlab/Simulink and experimental results are presented for the validity of the proposed control technique.
\end{abstract}

Keywords: VBC, DPS, Voltage bus conditioner, Bidirectional converter, PI compensator, Distributed power system.

\section{Introduction}

DC Distributed Power System(DPS)s have been widely used for communication systems, military VHSIC systems, bulky power supply systems, aircraft/aerospace, electric/hybrid car, regenerative energy systems, etc [1]-[5]. The general structures of the DC DPS are shown in Fig. 1. Most DC DPS architectures include an intermediate distribution bus, and are configured using various combination of cascading, load splitting, and so on.

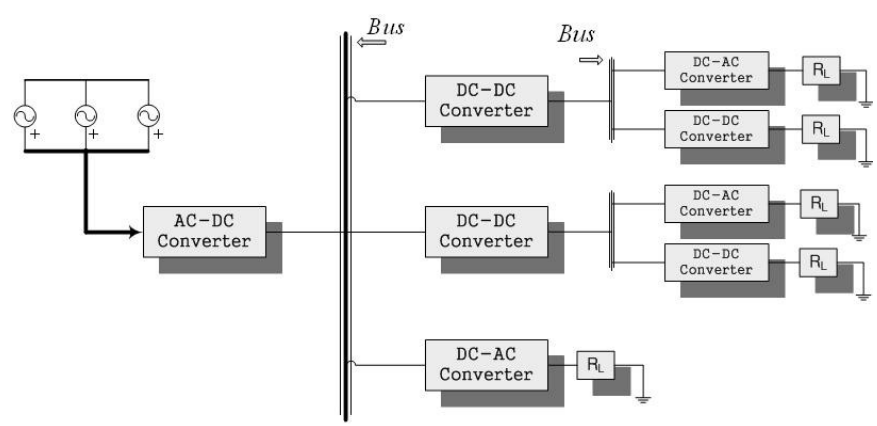

Fig. 1. General architecture of the DC distributed power system

In the DC DPS, the converter is required by the suitable filter due to the switching noise. Also, the converters have

* Dept. of Electrical Engineering, Inha University, Korea. ( joonminlee82@gmail.com)

* Dept. of Electrical Engineering, Inha University, Korea. (youngsk@inha.ac.kr)

** Dept. of Power Solution, Dawonsys Korea . (bongjoon9@naver.com)

*** Dept. of Electrical Engineering, Inha Technical University, Korea. (jenadu@gmail.com)

Received ; 13 July 2012; Accepted 30 July 2012
Constant Power Load (CPL) characteristics which lead to negative impedance [6]. In part, a DC DPS has a source converter and a load converter (Fig. 2).

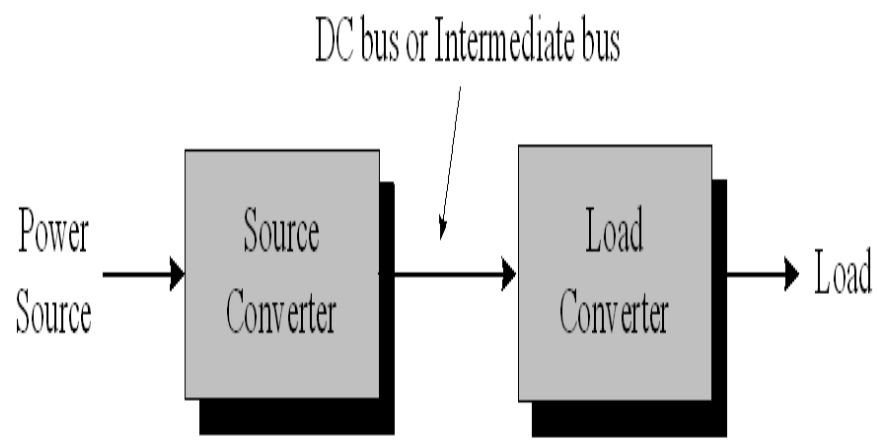

Fig. 2. Typical structure of a DC DPS

In Fig. 2, instead of the load converter, the load can be connected to the motor which consists of the Permanent Magnet Synchronous Motor (PMSM) and an Induction Motor (IM). The load with Constant Power Load (CPL) characteristics causes the negative impedance characteristics which might lead to the instability of the DC DPS due to interaction of the input filter [6]-[7].

The Voltage Bus Conditioner (VBC) is a bidirectional DC-DC converter for damping bus transient [8]-[10]. VBCs can be divided as storage types that are either the inductor type or the capacitor type. A VBC with the storage inductor type has the advantage of effectively using energy. However, under steady-state, the VBC causes a high level of power loss in the inductor. Also, if the load current level is higher than the inductor current level, the VBC could not been effectively regulated [8].

A VBC with the storage capacitor type avoids the above disadvantage. However, the bus voltage is always smaller 
than the storage voltage level [9]. Thus, the VBC is required by the larger capacitance. To avoid the above disadvantage, a VBC with a reduced capacitor has been proposed [10].

In this paper, the VBC with a reduced capacitor confirms the effectiveness that is necessary for the PI compensator. PI control can improve the steady-state error [11]. The locations of the zero and pole have to be selected properly so that the transient response is satisfactory.

\section{Bidirectional DC-DC Converter}

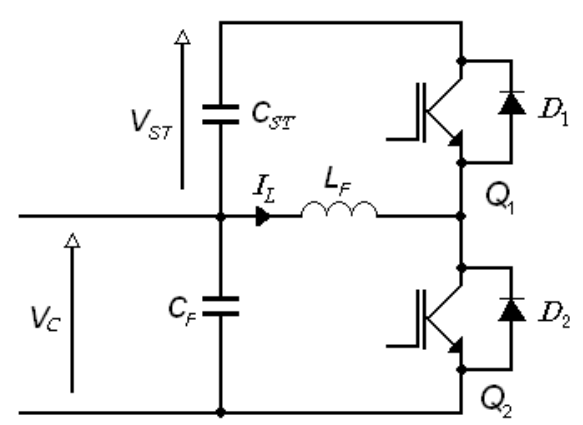

Fig. 3. Topology of the Voltage Bus Conditioner

The topology of the VBC is shown in Fig 3. In Fig 3, $V_{c}$ is the bus voltage, and $V_{S T}$ is the storage voltage. The filter capacitor $C_{F}$ is used for damping the switching ripple while both the switches are turned on or turned off. The inductor $L_{F}$ limits the current between the switches. $C_{S T}$ is the storage capacitor with the restored the energy during steady-state, D of the switching duty ratio can be defined as:

$$
D=\frac{V_{S T}}{V_{S T}+V_{C}}
$$

where, the value of the duty ratio $\mathrm{D}$ is determined by the switch $Q_{2}$.

\section{Averaged small-signal model}

The converters with switches can predict the converter characteristics from the state-space averaged model [12]. In Fig. 3, $I_{C}$ has the approximately value of zero. Thus, if $I_{C}=0, I_{S}$ is the same as $I_{L}$. When $I_{C}$ is zero, the state-space averaged model for a VBC is shown below,

$$
\left[\begin{array}{c}
\dot{\bar{V}}_{S T} \\
\dot{\bar{I}}_{L}
\end{array}\right]=\left[\begin{array}{cc}
0 & \frac{1-D}{C_{S T}} \\
-\frac{1-D}{L_{F}} & 0
\end{array}\right]\left[\begin{array}{c}
\bar{V}_{S T} \\
\bar{I}_{L}
\end{array}\right]+\left[\begin{array}{c}
0 \\
\frac{D}{L_{F}}
\end{array}\right] \bar{V}_{C}
$$

where the bars indicate the averaged value and $\mathrm{D}$ is the duty ratio of the bottom switch $Q_{2}$. The resulting linearized small signal model is:

$$
\left[\begin{array}{c}
\widetilde{V}_{S T} \\
\widetilde{I}_{L}
\end{array}\right]=\frac{\left[\begin{array}{c}
\frac{(1-D) D}{L_{F} C_{S T}} \\
\frac{D}{L_{F}} S
\end{array}\right]\left[\begin{array}{c}
\widetilde{V}_{S T} \\
\widetilde{I}_{L}
\end{array}\right]+\left[\begin{array}{l}
-\frac{I_{L}}{C_{S T}}-\frac{(1-D) V_{T}}{L_{F} C_{S T}} \\
\frac{V_{T}}{L_{F}} S+\frac{(1-D) I_{L}}{L_{F} C_{S T}}
\end{array}\right]}{s^{2}+\frac{(1-D)^{2}}{L_{F} C_{S T}}}
$$

where the tildes indicate small-signal quantities and $\mathrm{s}$ is the Laplace operator. The transfer function $G(s)$ of $\widetilde{I}_{L}$ over $\widetilde{D}$ can be extracted from (3) as:

$$
G(s)=\frac{\widetilde{I}_{L}}{\widetilde{D}}=\frac{\frac{V_{T}}{L_{F}} s+\frac{(1-D) I_{L}}{L_{F} C_{S T}}}{s^{2}+\frac{(1-D)^{2}}{L_{F} C_{S T}}}
$$

where $\bar{V}_{T}$ is $\bar{V}_{C}+\bar{V}_{S T}$. Under the steady-state switching, while both of the switches are operated, the value of $I_{L}$ has a negative or positive value. Thus, Right Half Plane (RHP) zero may occur. A system with a RHP zero is referred to as a non-minimum phase system [11]. Therefore, a $\mathrm{VBC}$ is required by the proper compensator.

\section{The proposed pi compensator}

\subsection{Transfer function of the Proportional Integration}

The Proportional Integration(PI) controller has the essentially characteristic of a Low Pass Filter(LPF), and the steady-state error of the original system improved by one order [11]. Generally, the transfer function of the PI controller is shown below,

$$
G(p)=\frac{K_{p}(s+P)}{s}
$$

where, $\mathrm{P}$ is $\frac{K_{1}}{K_{p}}$. The phase of the PI compensator is always negative, which is not desirable to the system stability. Thus, the corner frequency $\omega=\frac{K_{/}}{K_{p}}$ of the PI controller should be placed as far to the left side.

\subsection{P Gain and I Gain Calculation}

The transfer function of the uncompensated VBC is shown in Fig. 4. The initial phase is zero and the phase margin is 90 degree. 


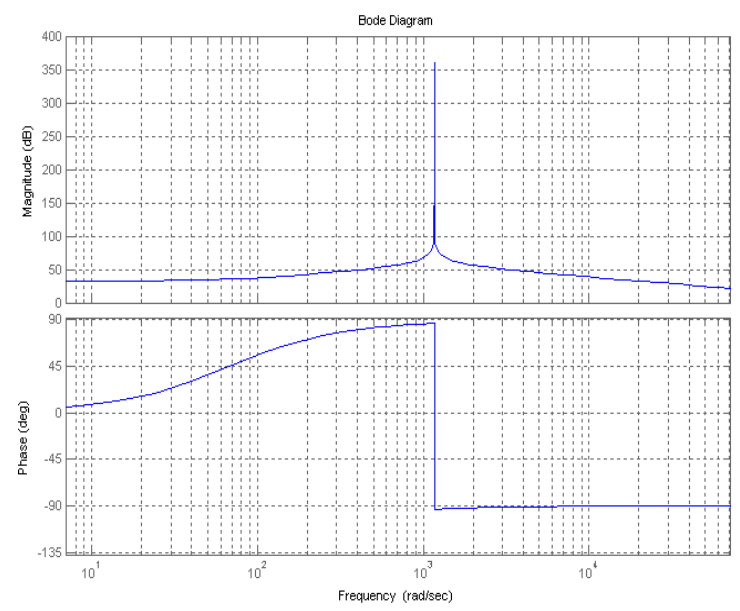

Fig. 4. Bode plot of the VBC

If the VBC is connected to the PI compensator, the new gain-crossover frequency $\omega_{g}^{\prime}$ corresponding to this phase margin is found on the bode plot. The magnitude plot of the compensated transfer function must pass through the $0-\mathrm{dB}$ axis. In order to obtain the desired phase, gains $\left(K_{P}, K_{I}\right)$ of the PI controller have to be chosen properly. Thus, $K_{P}$ has to be satisfied with the equation (6),

$$
K_{P}=10^{-\left|G_{P}\left(j \omega_{g}^{\prime}\right)\right|_{d B} / 20} \quad K_{P}<1
$$

After the value of $K_{P}$ is determined, it is necessary only to choose the proper value of $K_{I}$ to complete the design. In this paper, the new gain-crossover frequency is chosen as $5 \mathrm{kHz}$ which is fourth times smaller than the switching frequency of the system. Thus, the value of $K_{P}$ that is used in the equation (6) is calculated as 0.04 . For the attenuation property of PI controller, generally, the range of $\frac{K_{I}}{K_{P}}$ must satisfy following the requirement [11].

$$
\frac{K_{I}}{K_{P}}=\frac{\omega_{g}^{\prime}}{10 \sim 20} \mathrm{rad} / \mathrm{sec}
$$

Where, $K_{I}<70$. Thus, the value of $K_{I}$ is chosen as 40 .

\subsection{System control block}

The control block diagram of system is shown in Fig. 5 . The control system consists of two control loops. The main control loop is to damp the transients or instability of the DC bus. Also, the auxiliary control loop is to maintain the storage voltage at the desired level. Due to $K_{1}>>K_{2}$, the level of the auxiliary loop can be negligible. Thus, the open-loop transfer function of the system is shown as below,

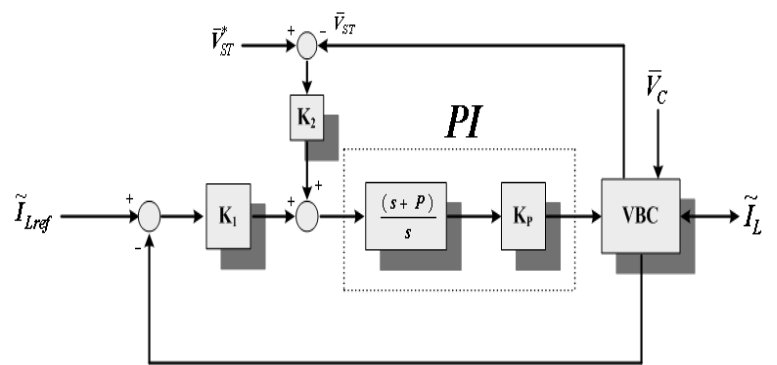

Fig. 5. Control block diagram of the VBC system

$$
G(s)=\frac{V_{T} K_{1} K_{P} C_{S T} \cdot s^{2}+\left(V_{T} P C_{S T}+(1-D) I_{L}\right) \cdot K_{P} K_{1} \cdot s+(1-D) P K_{P} K_{1} I_{L}}{L_{F} C_{F} \cdot s^{3}+(1-D)^{2} \cdot s}
$$

The bode plot of equation (8) is shown in Fig. 5.

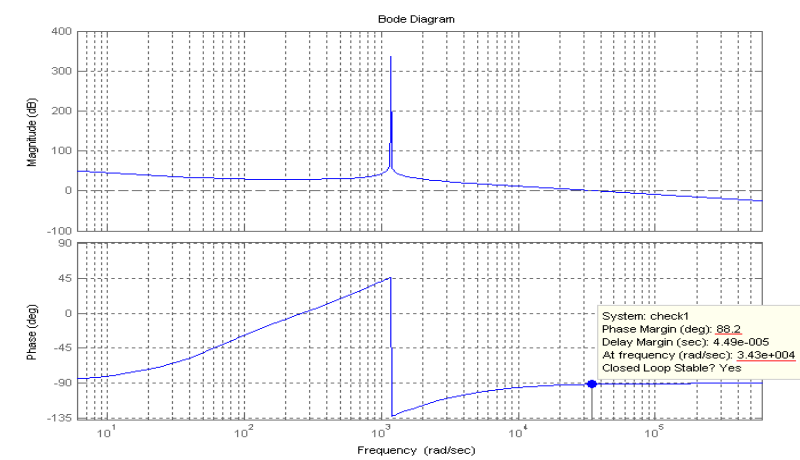

Fig. 6. Open-loop Bode plot of the VBC with PI compensator

The VBC with PI compensator has bandwidth $5 \mathrm{kHz}$, the phase margin is 88.2 degree. The bode plot of the closedloop transfer function is shown in Fig. 5. In Fig. 5, the damping ratio $\zeta$ is approximately 0.7 [13]. The system response has the slightly overshoot and a proper steadystate error. Thus, $K_{P}$ and $K_{I}$ of the PI compensator are chosen properly. The control block diagram of the overall system can be expressed in Fig. 8 .

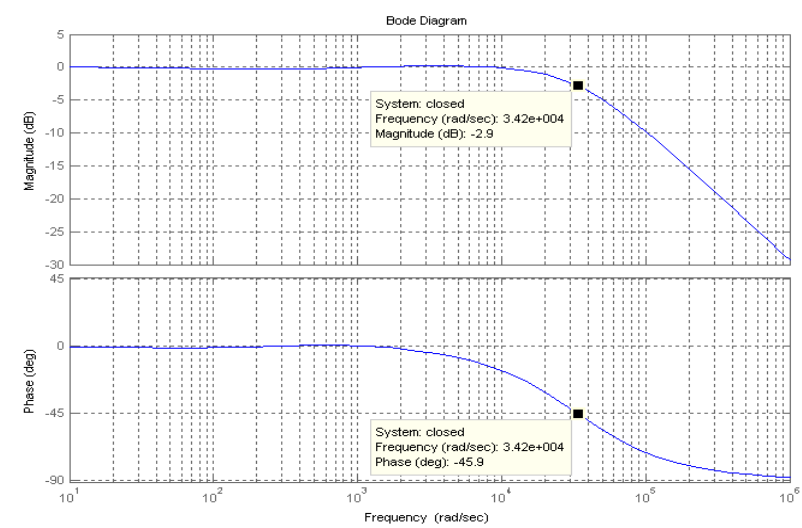

Fig. 7. Closed-loop Bode plot of the VBC with PI compensator 


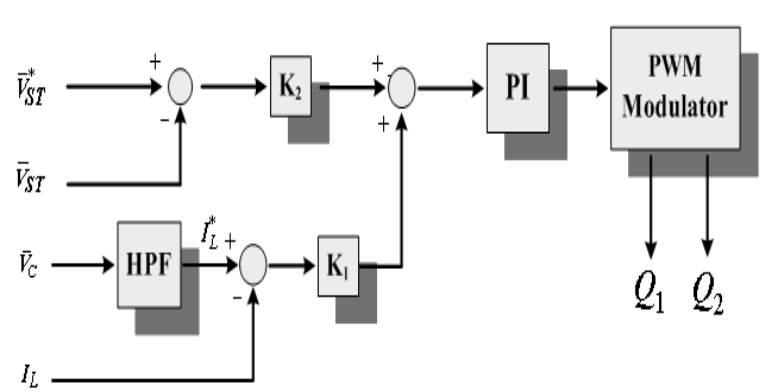

Fig. 8. Overall control blocks of the VBC system

The reference current $I_{L}^{*}$ is estimated from DC voltage by the High-Pass Filter (HPF). In the HPF, the cut-off frequency of the HPF was chosen as $1 \mathrm{kHz}$ by trial and error through simulations. The gain $K_{1}$ of the main control loop was chosen as 1 and the gain $K_{2}$ of the auxiliary control loop was chosen as 0.06 .

\section{Simulation and Experimental results}

The VBC with the reduced storage capacitor is connected to the DC bus in the distributed power system. The overall system has been simulated by MATLAB/Simulink. The block diagram of the system is shown in Fig 9.

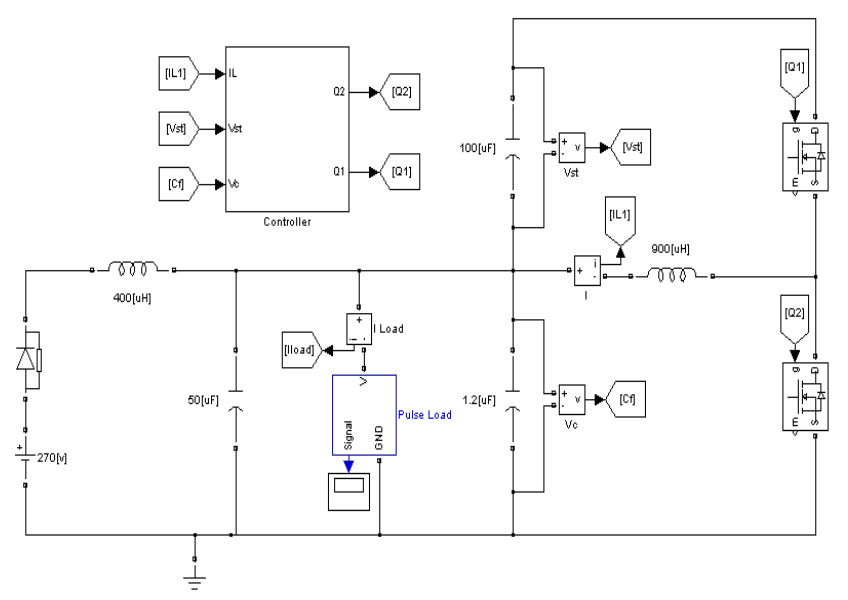

Fig. 9. Block diagram of system using Matlab Simulink

The system parameters are shown in the Table I. The pulsed load is a resistive load which bring $10 \mathrm{~A}$ from the bus voltage in the steady-state.

Table 1. Circuit parameters of the simulation

\begin{tabular}{|c|c|c|}
\hline Parameter & Symbol & Value \& Unit \\
\hline Bus Voltage & $V_{C}$ & $200 \mathrm{~V}$ \\
\hline Inductance of LC Filter & $L_{S}$ & $400 \mu \mathrm{H}$ \\
\hline
\end{tabular}

\begin{tabular}{|c|c|c|}
\hline Capacitance of LC Filter & $C_{S}$ & $50 \mu \mathrm{F}$ \\
\hline Storage capacitor of the VBC & $C_{S T}$ & $100 \mu \mathrm{F}$ \\
\hline Inductance Of the VBC & $L_{F}$ & $900 \mu \mathrm{H}$ \\
\hline Capacitance of the VBC & $C_{F}$ & $1.2 \mu \mathrm{F}$ \\
\hline Rated Pulsed Load & - & $2 \mathrm{~kW}$ \\
\hline Switching frequency & - & $20 \mathrm{kHz}$ \\
\hline
\end{tabular}

The simulation results when the resistive load is connected are shown in Fig. 10. In Fig. 10(a), without the VBC (dotted line), the bus voltage variations are between $174 \mathrm{~V}$ and $224.5 \mathrm{~V}$. Then the oscillation frequency occurs at approximately $1.1 \mathrm{kHz}$ by the input filter $L_{S}$ and $C_{S}$. With VBC (a solid line), the bus voltage variations are between $180 \mathrm{~V}$ and $222.5 \mathrm{~V}$. Also, the oscillations are damped within around $1.5 \mathrm{msec}$. The storage voltage level is averagely maintained at $360 \mathrm{~V}$ (Fig. 10(c)).
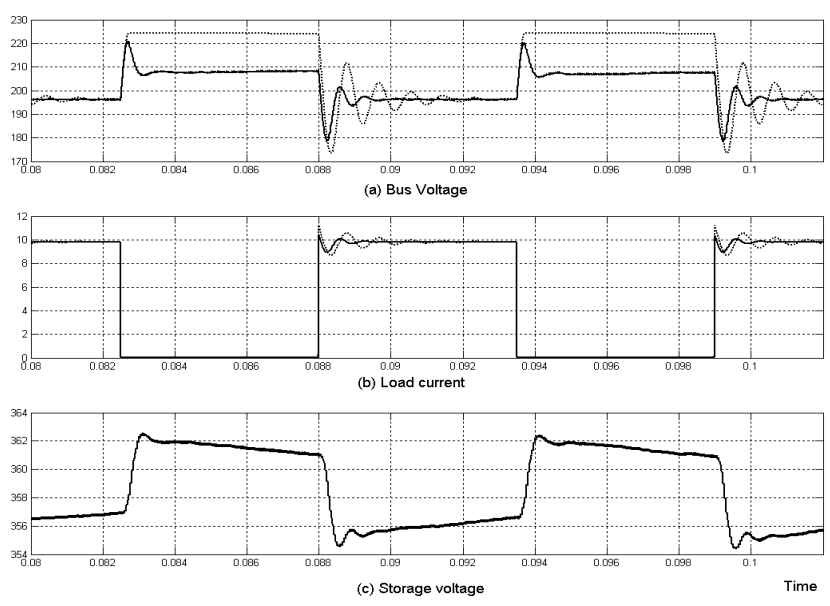

Fig. 10. Simulation results for the resistive load

The experimental parameters are shown in the Table II.The pulsed load is a resistive load which brings around $10 \mathrm{~A}$ from the bus voltage in the steady-state.

Table 2. Circuit parameters of the experiment

\begin{tabular}{|c|c|c|}
\hline Parameter & Symbol & Value \& Unit \\
\hline Bus Voltage & $V_{C}$ & $200 \mathrm{~V}$ \\
\hline Inductance of LC Filter & $L_{S}$ & $401 \mu \mathrm{H}$ \\
\hline Capacitance of LC Filter & $C_{S}$ & $49.2 \mu \mathrm{F}$ \\
\hline Storage capacitor of the VBC & $C_{S T}$ & $94.7 \mu \mathrm{F}$ \\
\hline Inductance Of the VBC & $L_{F}$ & $901 \mu \mathrm{H}$ \\
\hline Capacitance of the VBC & $C_{F}$ & $1 \mu \mathrm{F}$ \\
\hline Rated Pulsed Load & - & $2 \mathrm{~kW}$ \\
\hline Switching frequency & - & $20 \mathrm{kHz}$ \\
\hline
\end{tabular}

The experiment results when the resistive load is connected are shown in Fig. 11 (without VBC). The bus voltage variations are between $176 \mathrm{~V}$ and $226 \mathrm{~V}$. In addition, the $1.14 \mathrm{kHz}$ oscillation frequency by the input 
filter components $\left(L_{S}\right.$ and $\left.C_{S}\right)$ can be seen. In Fig. 12 (with VBC), the bus voltage variations are between $180 \mathrm{~V}$ and $222.5 \mathrm{~V}$. The oscillations are damping within $1.5 \mathrm{msec}$. In Fig. 13, the average storage voltage is just below $360 \mathrm{~V}$.

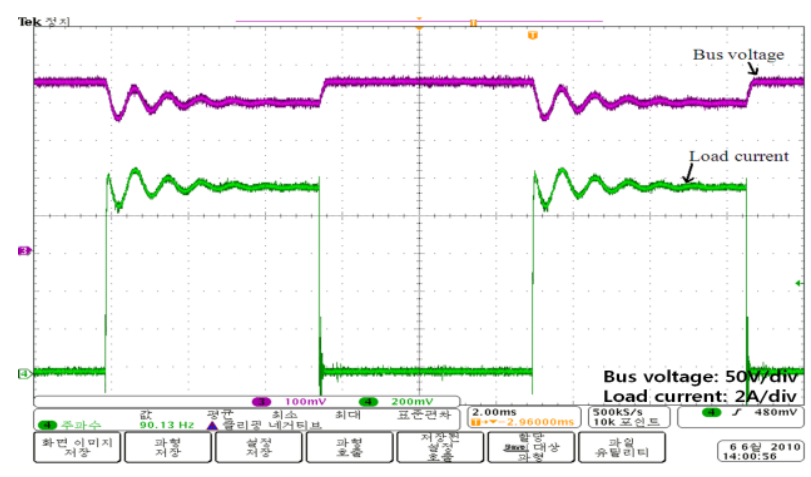

Fig. 11. Experimental results for the resistive load without VBC

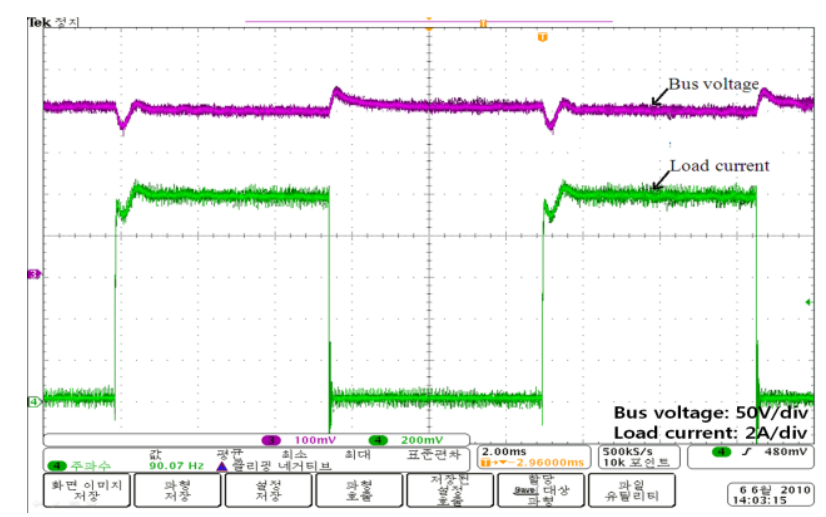

Fig. 12. Experimental results for the resistive load with VBC

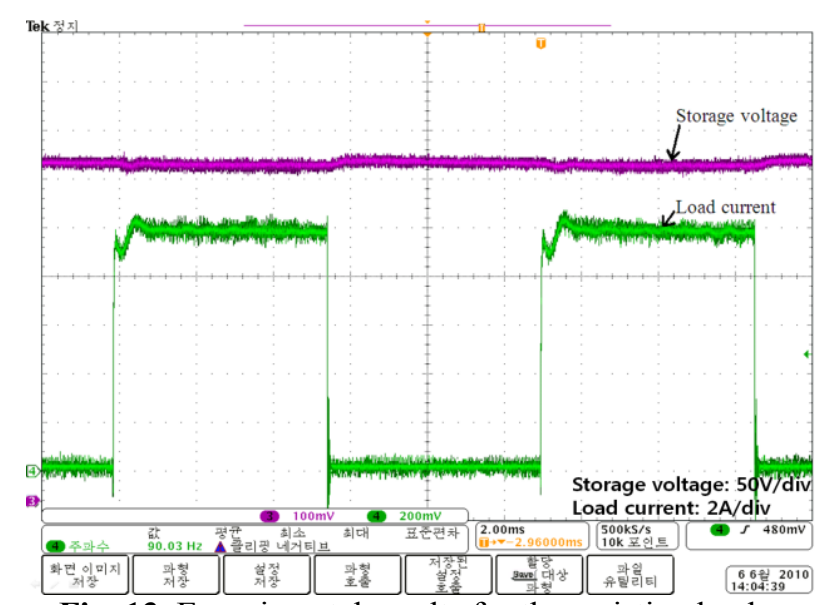

Fig. 13. Experimental results for the resistive load with VBC
This paper has presented the VBC system with the proposed PI compensator with constant switching frequency. The simulation by Matlab Simulink and experiment result show that the proposed approach not only effectively mitigates the bus voltage transients caused by pulsed power loads, but also contributes to the improvement of the power quality and stability of the power distribution system.

\section{Acknowledgements}

This research was supported by the MKE(The Ministry of Knowledge Economy), Korea, under the ITRC(Information Technology Research Center) support program supervised by the NIPA(National IT Industry Promotion Agency) (NIPA-2012-C1090-1200-0007)

\section{References}

[1] C. C. Heath, "The Market for Distributed Power System", Applied Power Electronics Conference and Exposition 1991, APEC'91, Conference Proceedings 1991, 6th Annual IEEE, Mar. 1991, Page(s) $225-229$

[2] W. A. Tabisz, M. M. Jovanovic, F. C. Lee, "Present and Future of Distributed Power Systems", Applied Power Electronics Conference and Exposition 1992, APEC'92, Conference Proceedings 1992, 7th Annual IEEE, Feb. 1992, Page(s) $11-18$.

[3] Jih-Sheng Lai, D.J. Nelson, "Energy Management Power Converters in Hybrid Electric and Fuel Cell Vehicles", Proceedings of the IEEE, Vol. 95. NO.4, April 2007

[4] S, Luo, "A Review of Distributed Power Systems, part I:DC Distributed Power System", aerospace and Electronic Systems Magazine, IEEE, Page(s) 5-16, Aug.

[5] Mammano,B. "Distributed Power Systems", In Proceedings of Unitrode Power Supply Design Seminar(SEM-900),1993,1-11.

[6] A. Emadi, A. Khakigh, C. H. Rivetta, G. A. Williamson, "Constant Power Loads and Negative Impedance Instability in Automotive System: Definition, Modeling, Stability, and Control of Power Electronics Converters and Motor Drives", IEEE Transactions on Vehicular Technology, Vol.55 NO.4, July 2006.

[7] M. Belkhayat, R. Cooley, A. Witulski, "Large Signal Stability Criteria For Distributed Systems with Constant Power Loads", in Proc. IEEE $26^{\text {th }}$ Power Electron. Spec. Conf., Atlanta, GA, Jun. 1995, pp. 13331338.

[8] S.V. Mollov, and J.D. LA, "Study of control algorithms for a voltage bus conditioner", Vehicle Power and Propulsion 2005 IEEE Conference, Page(s) 372 - 378, SEP 2005

[9] Kun Xing, Jinhong Guo, Wenkang Huang, Dengming Peng, Fred C. Lee, Dusan Borjevic Center for Power Electronics System, "An Active Bus Conditioner for a Distributed Power System, Electronics Specialists Conference, Vol.2, pager(s) 895-900, July 1999

[10] J.D La, "An Active Damping Device for a Distributed Power System", The transaction of KIEE. v.58p, no.2, pp.116-121, June. 2009.

[11] B.C. Kuo, "Automatic Control Systems", 8th edition , John Wiley \& Sons, 2006.

[12] Forsyth, A.J. Mollov, S.V. , "Modelling and control of DC-DC converters" , IEEE Power Engineering Journal, pp:229-236 , Oct 1998

[13] K. Ogata, "Modern Control Engineering", $4^{\text {th }}$ edition, Prentice- Hall inc. , 2002

\section{Conclusion}



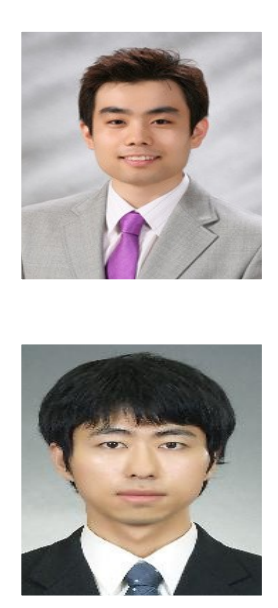

Joonmin Lee received his Master degree in Electrical Engineering from Inha university. His research interests are power electronics, electric machines, control technique of electric motors and renewable energy systems.

Bong Jun Seok received his Master degree in Electrical Engineering from Inha university. His research interests are power electronics, all-electric aircraft and power conditioning systems.

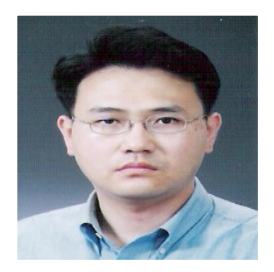

Jae Du La received his Ph.D degree in Electrical Engineering from the University of Birmingham (UK). He is an assistant professor in the department of Electrical Information, Inha Technical College, Incheon, South Korea, 2003 - Present. His research interests are power electronics, more and all-electric aircraft, power conditioning systems and renewable energy system

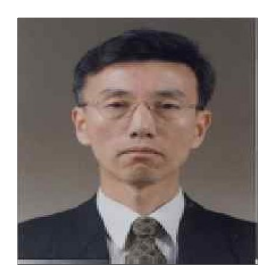

Young Seok Kim received his Ph.D degree in Electrical Engineering from Nagoya university(Japan). $\mathrm{He}$ is a professor in the department of Electrical Engineering, Inha University, Incheon, South Korea, 1989 - Present.

His research interests are power electronics, electric machines \& control technique of electric motors and renewable energy systems 